\title{
A New Asymptotic Series and Estimates Related to Euler Mascheroni Constant
}

\author{
VALENTIN GABRIEL CRISTEA*
}

ABSTRACT. In this article, we give a new asymptotic series for a sequence $\left(q_{n}\right)$ that converges to Euler-Mascheroni's constant with the convergence speed as $n^{-4}$. We present and prove a theorem about how to get the sequence $\left(q_{n}\right)$. Using this asymptotic series, we establish the lower and upper bounds for the sequence $\left(q_{n}\right)$.

Keywords: Euler-Mascheroni's constant, asymptotic series, inequalities.

2010 Mathematics Subject Classification: 26D15, 41A25, 34E05.

\section{INTRODUCTION}

One of the famous constants in mathematics is the Euler-Mascheroni's constant $\gamma=0,57721566490153286 \ldots$. It is defined as the limit of the sequence:

$$
\gamma_{n}=1+\frac{1}{2}+\frac{1}{3}+\ldots+\frac{1}{n}-\ln n
$$

in honor of the Swiss mathematician Leonhard Euler (1707-1783) and the Italian mathematician Lorenzo Mascheroni (1750-1800), who studied the Euler-Mascheroni's constant $\gamma$. The sequence $\left(\gamma_{n}\right)_{n \geq 1}$ and the constant $\gamma$ have many applications in several branches of mathematics as probability, analysis, special functions and number theory. The sequence $\left(\gamma_{n}\right)_{n \geq 1}$ converges very slowly to the constant $\gamma$, with the convergence speed as $n^{-1}$. In the beginning, Tims and Tyrell [18], and then Young [19] got the lower and upper bounds for the sequence $\left(\gamma_{n}\right)_{n \geq 1}$ as the following:

$$
\frac{1}{2(n+1)}<\gamma_{n}-\gamma<\frac{1}{2 n}
$$

with the convergence speed as $n^{-1}$. Many authors [2,3,6,7,10,12-17] interested in obtaining sequences that converge very fast to the limit $\gamma$. One of them is DeTemple [6], who introduced the sequence

$$
R_{n}=1+\frac{1}{2}+\frac{1}{3}+\ldots+\frac{1}{n}-\ln \left(n+\frac{1}{2}\right)
$$

that converges to the limit $\gamma$ as $n^{-2}$. Then Mortici [12] has introduced the sequence

$$
t_{n}=1+\frac{1}{2}+\ldots+\frac{1}{n-1}+\frac{1}{2 n}-\frac{1}{2} \ln \left(n^{2}-\frac{1}{6}\right)
$$

Received: 21.08.2019; Accepted: 23.11.2019; Published Online: 02.01.2020

*Corresponding author: Valentin Gabriel Cristea; valentingabrielc@yahoo.com

DOI:10.33205/cma.608927 
in order to obtain a faster convergence to the limit $\gamma$ with the convergence speed as $n^{-4}$ and the following limit:

$$
\lim _{n \rightarrow \infty} n^{4}\left(t_{n}-\gamma\right)=\frac{11}{720}
$$

Then, Cristea [4] has showed in 2014, the following double inequality

$$
\frac{11}{720 n^{4}}-\frac{29}{9072 n^{6}}<t_{n}-\gamma<\frac{11}{720 n^{4}}
$$

for all integers $n \geq 1$ and has got the following asymptotic series for the sequence $\left(t_{n}\right)$ given in (1.1)

or

$$
t_{n}=\gamma+\sum_{k=2}^{\infty} \frac{1}{2 k}\left\{\frac{1}{6^{k}}-B_{2 k}\right\} \frac{1}{n^{2 k}}
$$

$$
t_{n}=\gamma+\frac{11}{720 n^{4}}-\frac{29}{9072 n^{6}}+\frac{221}{51840 n^{8}}-\frac{6469}{855360 n^{10}}+\cdots
$$

Cristea and Mortici [5] have introduced the sequence

$$
s_{n}=1+\frac{1}{2}+\ldots+\frac{1}{n-2}+\frac{13}{12(n-1)}+\frac{5}{12 n}-\ln n
$$

that converges to the limit $\gamma$ with the convergence speed as $n^{-3}$ and have demonstrated the following double inequality

$$
\frac{1}{12 n^{3}}+\frac{11}{120 n^{4}}<s_{n}-\gamma<\frac{1}{12 n^{3}}+\frac{13}{120 n^{4}} .
$$

Then, X. Hu, D. Lu, X. Wang [9] have presented the following sequence:

$$
r_{n, 2}^{3}=1+\frac{1}{2}+\ldots+\frac{1}{n}-\ln n-\frac{1}{2} \ln \left(1+\frac{1}{n-\frac{n}{3 n+1}}\right)
$$

that converges to the limit $\gamma$ with the convergence speed as $n^{-4}$, with the following approximation:

$$
\frac{1}{180(n+1)^{4}}<\gamma-r_{n, 2}^{3}<\frac{1}{180 n^{4}} .
$$

The aim of the paper is to introduce a new sequence $\left(q_{n}\right)$ that converges very fast to the limit $\gamma$ and to establish the lower and upper bounds for this sequence. Motivated by Mortici [12] and $\mathrm{Hu}[9]$, we introduce new sequence

$$
q_{n}(a, b, c)=1+\frac{1}{2}+\ldots+\frac{1}{n-2}+\frac{a n+b}{n(n-1)}-\frac{1}{3} \ln \left(n^{3}+c\right),
$$

where $a, b, c$ are real parameters and for $a=\frac{3}{2}, b=-\frac{5}{12}, c=\frac{1}{4}$ the new sequence given by

$$
q_{n}=q_{n}\left(\frac{3}{2},-\frac{5}{12}, \frac{1}{4}\right)=1+\frac{1}{2}+\ldots+\frac{1}{n-2}+\frac{13}{12(n-1)}+\frac{5}{12 n}-\frac{1}{3} \ln \left(n^{3}+\frac{1}{4}\right)
$$

converges to the limit $\gamma$ with the convergence speed as $n^{-4}$. We will show the following double inequality

$$
\frac{11}{120 n^{4}}+\frac{1}{12 n^{5}}+\frac{181}{2016 n^{6}}<q_{n}-\gamma<\frac{11}{120 n^{4}}+\frac{1}{12 n^{5}}+\frac{182}{2016 n^{6}}
$$

for all integers $n \geq 2$ in the left side inequality and for all integers $n \geq 225$ in the right side inequality. We will also construct the asymptotic series

$$
q_{n}=\gamma+\frac{11}{120 n^{4}}+\frac{1}{12 n^{5}}+\frac{181}{2016 n^{6}}+\frac{1}{12 n^{7}}+\cdots
$$


for the sequence $\left(q_{n}\right)(1.4)$.

\section{The Results}

We consider the sequence $\left(q_{n}(a, b, c)\right)$ given by (1.3). To obtain the best real parameters $a, b, c$, for which the sequence $\left(q_{n}(a, b, c)\right)$ converges to $\gamma$ with the highest convergence speed, we prove the following theorem:

Theorem 2.1. (i) If $a \neq \frac{3}{2}, b \neq-\frac{5}{12}$ and $c \neq \frac{1}{4}$ then the sequence $\left(q_{n}(a, b, c)\right)_{n \geq 1}$ has the convergence speed as $n^{-1}$.

(ii) If $a=\frac{3}{2}, b \neq-\frac{5}{12}$ and $c \neq \frac{1}{4}$ then the sequence $\left(q_{n}(a, b, c)\right)_{n \geq 1}$ has the convergence speed as $n^{-2}$.

(iii) If $a=\frac{3}{2}, b=-\frac{5}{12}$ and $c \neq \frac{1}{4}$ then the sequence $\left(q_{n}(a, b, c)\right)_{n \geq 1}$ has the convergence speed as $n^{-3}$.

(iv) If $a=\frac{3}{2}, b=-\frac{5}{12}$ and $c=\frac{1}{4}$ then the sequence $\left(q_{n}(a, b, c)\right)_{n \geq 1}$ has the convergence speed as $n^{-4}$.

We will use the following:

Lemma 2.1. If the sequence $\left(x_{n}\right)_{n \geq 1}$ converges to $x$ and if there exists the limit

$$
\lim _{n \rightarrow \infty} n^{k}\left(x_{n}-x_{n+1}\right)=l \in \mathbb{R}
$$

with $k>1$, then there exists the limit

$$
\lim _{n \rightarrow \infty} n^{k-1}\left(x_{n}-x\right)=\frac{l}{k-1} .
$$

For the proof see [11]. This lemma is a form of Cesaro-Stolz's lemma. We utilize it in the construction of the asymptotics series and in order to estimate the convergence speed.

Proof. We compute the difference

$$
\begin{gathered}
q_{n}(a, b, c)-q_{n+1}(a, b, c)=\frac{a n+b}{n(n-1)}-\frac{1}{n-1}-\frac{a n+a+b}{n(n+1)} \\
-\frac{1}{3} \ln \left(n^{3}+c\right)+\frac{1}{3} \ln \left((n+1)^{3}+c\right) .
\end{gathered}
$$

Using a computer program as Maple, we get

$$
\begin{gathered}
q_{n}(a, b, c)-q_{n+1}(a, b, c)=\left(a-\frac{3}{2}\right) \frac{1}{n^{2}}+\left(a+2 b-\frac{2}{3}\right) \frac{1}{n^{3}}+\left(a-c-\frac{5}{4}\right) \frac{1}{n^{4}} \\
+\left(a+2 b+2 c-\frac{4}{5}\right) \frac{1}{n^{5}}+O\left(\frac{1}{n^{6}}\right) .
\end{gathered}
$$

(i) If $a-\frac{3}{2} \neq 0$, then

$$
\lim _{n \rightarrow \infty} n^{2}\left(q_{n}(a, b, c)-q_{n+1}(a, b, c)\right)=\left(a-\frac{3}{2}\right) \neq 0
$$

and Lemma 2.1 says that

$$
\lim _{n \rightarrow \infty} n\left(q_{n}(a, b, c)-\gamma\right)=\left(a-\frac{3}{2}\right) \neq 0 .
$$


We get that the sequence $\left(q_{n}(a, b, c)\right)_{n>1}$ has the convergence speed as $n^{-1}$.

(ii) If $a=\frac{3}{2}, b \neq-\frac{5}{12}$ and $c \neq \frac{1}{4}$ then the relation (2.5) is written as

$$
\begin{gathered}
q_{n}(a, b, c)-q_{n+1}(a, b, c)=\left(2 b+\frac{5}{6}\right) \frac{1}{n^{3}}+\left(\frac{1}{4}-c\right) \frac{1}{n^{4}} \\
+\left(\frac{7}{10}+2 b+2 c\right) \frac{1}{n^{5}}+O\left(\frac{1}{n^{6}}\right) .
\end{gathered}
$$

If $b \neq-\frac{5}{12}$, then from the relation (2.6), we get

$$
\lim _{n \rightarrow \infty} n^{3}\left(q_{n}(a, b, c)-q_{n+1}(a, b, c)\right)=\left(2 b+\frac{5}{6}\right) \neq 0
$$

and Lemma 2.1 says that

$$
\lim _{n \rightarrow \infty} n^{2}\left(q_{n}(a, b, c)-\gamma\right)=\frac{1}{2}\left(2 b+\frac{5}{6}\right) \neq 0 .
$$

We obtain that the sequence $\left(q_{n}\left(\frac{3}{2}, b, c\right)\right)_{n \geq 1}$ has the convergence speed as $n^{-2}$.

(iii) If $a=\frac{3}{2}, b=-\frac{5}{12}$ and $c \neq \frac{1}{4}$ then the relation (2.5) is written as

$$
q_{n}(a, b, c)-q_{n+1}(a, b, c)=\left(\frac{1}{4}-c\right) \frac{1}{n^{4}}+\left(-\frac{2}{15}+2 c\right) \frac{1}{n^{5}}+O\left(\frac{1}{n^{6}}\right) .
$$

Then from the relation (2.7), we get

$$
\lim _{n \rightarrow \infty} n^{4}\left(q_{n}(a, b, c)-q_{n+1}(a, b, c)\right)=\left(\frac{1}{4}-c\right) \neq 0
$$

and Lemma 2.1 says that

$$
\lim _{n \rightarrow \infty} n^{3}\left(q_{n}(a, b, c)-\gamma\right)=\frac{1}{3}\left(\frac{1}{4}-c\right) \neq 0 .
$$

We get that the sequence $\left(q_{n}\left(\frac{3}{2},-\frac{5}{12}, c\right)\right)_{n \geq 1}$ has the convergence speed as $n^{-3}$.

(iv) If $a=\frac{3}{2}, b=-\frac{5}{12}$, and $c=\frac{1}{4}$ then the relation (2.5) is written as

$$
q_{n}(a, b, c)-q_{n+1}(a, b, c)=\frac{11}{30 n^{5}}+O\left(\frac{1}{n^{6}}\right)
$$

and Lemma 2.1 says that

$$
\lim _{n \rightarrow \infty} n^{4}\left(q_{n}(a, b, c)-\gamma\right)=\frac{11}{120} .
$$

We get that the sequence $\left(q_{n}\left(\frac{3}{2},-\frac{5}{12}, \frac{1}{4}\right)\right)_{n \geq 1}$ has the convergence speed as $n^{-4}$.

We notice that (2.8) gives us the approximation

$$
q_{n}-\gamma \approx \frac{11}{120 n^{4}} \text { as } n \rightarrow \infty
$$

We give the following theorem related to the estimates of $\left(q_{n}\right)$ given in (1.4):

Theorem 2.2. We have the following double inequality for all integers $n \geq 2$ in the left side inequality and for all integers $n \geq 225$ in the right side inequality:

$$
\frac{11}{120 n^{4}}+\frac{1}{12 n^{5}}+\frac{181}{2016 n^{6}}<q_{n}-\gamma<\frac{11}{120 n^{4}}+\frac{1}{12 n^{5}}+\frac{182}{2016 n^{6}} .
$$


Proof. We consider the following sequences

$$
a_{n}=\left(q_{n}-\gamma\right)-\left(\frac{11}{120 n^{4}}+\frac{1}{12 n^{5}}+\frac{181}{2016 n^{6}}\right)
$$

and

$$
b_{n}=\left(q_{n}-\gamma\right)-\left(\frac{11}{120 n^{4}}+\frac{1}{12 n^{5}}+\frac{182}{2016 n^{6}}\right)
$$

that converges to zero. To prove that $a_{n}>0$ and $b_{n}<0$, it suffices to show that $\left(a_{n}\right)_{n \geq 1}$ is strictly decreasing and $\left(b_{n}\right)_{n \geq 1}$ is strictly increasing. Let $f_{1}(n)=a_{n+1}-a_{n}$ and $f_{2}(n) \stackrel{=}{=}$ $b_{n+1}-b_{n}$, where

$$
\begin{aligned}
f_{1}(x)= & \frac{8}{12 x}+\frac{5}{12(x+1)}-\frac{1}{12(x-1)}+\frac{1}{3} \ln \left(x^{3}+\frac{1}{4}\right)-\frac{1}{3} \ln \left((x+1)^{3}+\frac{1}{4}\right) \\
& -\left(\frac{11}{120(x+1)^{4}}-\frac{11}{120 x^{4}}\right)-\left(\frac{1}{12(x+1)^{5}}-\frac{1}{12 x^{5}}\right)-\left(\frac{181}{2016(x+1)^{6}}-\frac{181}{2016 x^{6}}\right)
\end{aligned}
$$

and

$$
\begin{aligned}
f_{2}(x)= & \frac{8}{12 x}+\frac{5}{12(x+1)}-\frac{1}{12(x-1)}+\frac{1}{3} \ln \left(x^{3}+\frac{1}{4}\right)-\frac{1}{3} \ln \left((x+1)^{3}+\frac{1}{4}\right) \\
& -\left(\frac{11}{120(x+1)^{4}}-\frac{11}{120 x^{4}}\right)-\left(\frac{1}{12(x+1)^{5}}-\frac{1}{12 x^{5}}\right)-\left(\frac{182}{2016(x+1)^{6}}-\frac{182}{2016 x^{6}}\right) .
\end{aligned}
$$

We get

$$
f_{1}^{\prime}(x)=\frac{P(x-2)}{1680(x+1)^{7}(x-1)^{2}\left(4 x^{3}+1\right)^{1}\left(12 x+12 x^{2}+4 x^{3}+5\right)^{1} x^{5}}>0
$$

for all real numbers $x \geq 2$ and

$$
f_{2}^{\prime}(x)=-\frac{Q(x-225)}{120(x+1)^{7}(x-1)^{2}\left(12 x+12 x^{2}+4 x^{3}+5\right)^{1}\left(4 x^{3}+1\right)^{1} x^{7}}<0
$$

for all real numbers $x \geq 225$, where

$$
\begin{aligned}
P(x)= & 8615781393+48322358535 x+124451770884 x^{2}+195088765300 x^{3} \\
& +207843366162 x^{4}+159018283386 x^{5}+89932803430 x^{6}+38082594545 x^{7} \\
& +12078804629 x^{8}+2834912752 x^{9}+478671564 x^{10}+55071128 x^{11} \\
& +3869824 x^{12}+125440 x^{13}
\end{aligned}
$$


and

$$
\begin{aligned}
Q(x)= & 22876348962124636919596278035200 \\
& +156125891834161825105090815353280 x \\
& +8964689205792820697567513156375 x^{2} \\
& +238298913583029626485888825003 x^{3} \\
& +3874001939229085395299660913 x^{4} \\
& +42953509800254866165809975 x^{5} \\
& +342954298088658683537331 x^{6} \\
& +2028513740325127816093 x^{7} \\
& +8999214295901801973 x^{8} \\
& +29943893833882652 x^{9} \\
& +73805584698144 x^{10} \\
& +130981721712 x^{11} \\
& +158491784 x^{12} \\
& +117200 x^{13} \\
& +40 x^{14}
\end{aligned}
$$

are two polynomials with positive integers coefficients for all real numbers $x \geq 2$ and respectively for all real numbers $x \geq 225$. Then, from (2.9), we have $f_{1}$ is strictly increasing on $[2, \infty)$ and from $(2.10)$, we have $f_{2}$ is strictly decreasing on $[225, \infty)$. It follows that from $f_{1}(\infty)=f_{2}(\infty)=0$, we have $f_{1}<0$ on $[2, \infty)$ and $f_{2}>0$ on $[225, \infty)$. Thus, $\left(a_{n}\right)_{n \geq 2}$ is strictly decreasing and $\left(b_{n}\right)_{n \geq 225}$ is strictly increasing. This concludes the proof.

We can get the asymptotic series of the sequence $\left(q_{n}\right)$, using the sequence $\left(h_{n}\right)$

$$
h_{n}=1+\frac{1}{2}+\ldots+\frac{1}{n-2}+\frac{1}{n-1}+\frac{1}{n}
$$

harmonic sum in terms of digamma function $\psi$

$$
h_{n}=\gamma+\frac{1}{n}+\psi(n),
$$

with the digamma function defined by

$$
\psi(x)=\frac{d}{d x}(\ln \Gamma(x))=\frac{\Gamma^{\prime}(x)}{\Gamma(x)} .
$$

See, e.g., [1, p. 258, Rel. 6.3.2]. We have the following asymptotic expansion for the digamma function $\psi$ that

$$
\psi(x)=\ln x-\frac{1}{2 x}-\sum_{k=1}^{\infty} \frac{B_{2 k}}{2 k x^{2 k}},
$$

where $B_{j}$ is the $j$ th Bernoulli numbers given by

$$
\frac{1}{e^{t}-1}+\frac{1}{2}-\frac{1}{t}=\sum_{j=1}^{\infty}(-1)^{j-1} \frac{t^{2 j}}{(2 j) !} B_{j} .
$$

We will demonstrate the following theorem related to the asymptotic expansion of $q_{n}$ : 
Theorem 2.3. We get the following asymptotic expansion of $\left(q_{n}\right)$ as $n \rightarrow \infty$ :

$$
q_{n}=\gamma+\frac{1}{12 n(n-1)}-\sum_{k=1}^{\infty} \frac{1}{k}\left\{\frac{(-1)^{k-1}}{3 \cdot 4^{k} n^{3 k}}+\frac{B_{2 k}}{2 n^{2 k}}\right\} .
$$

Proof. We get

$$
\begin{aligned}
q_{n} & =h_{n}-\frac{1}{n}+\frac{1}{12(n-1)}+\frac{5}{12 n}-\frac{1}{3} \ln \left(n^{3}+\frac{1}{4}\right) \\
& =\gamma+\psi(n)+\frac{1}{12(n-1)}+\frac{5}{12 n}-\frac{1}{3} \ln \left(n^{3}+\frac{1}{4}\right) \\
& =\gamma+\psi(n)-\ln n+\frac{1}{12(n-1)}+\frac{5}{12 n}-\frac{1}{3} \ln \left(1+\frac{1}{4 n^{3}}\right) \\
& =\gamma+\frac{1}{12(n-1)}-\frac{1}{2 n}+\frac{5}{12 n}-\sum_{k=1}^{\infty} \frac{B_{2 k}}{2 k n^{2 k}}-\frac{1}{3} \ln \left(1+\frac{1}{4 n^{3}}\right) \\
& =\gamma+\frac{1}{12 n(n-1)}-\sum_{k=1}^{\infty} \frac{1}{k}\left\{\frac{(-1)^{k-1}}{3 \cdot 4^{k} n^{3 k}}+\frac{B_{2 k}}{2 n^{2 k}}\right\} .
\end{aligned}
$$

Using the binomial theorem given in [8], we get

$$
\frac{1}{12 n(n-1)}=\frac{1}{12 n^{2}\left(1-\frac{1}{n}\right)}=\frac{1}{12 n^{2}}+\frac{1}{12 n^{3}}+\frac{1}{12 n^{4}}+\frac{1}{12 n^{5}}+\cdots
$$

We get an explicite form as

$$
q_{n}=\gamma+\frac{11}{120 n^{4}}+\frac{1}{12 n^{5}}+\frac{181}{2016 n^{6}}+\frac{1}{12 n^{7}}+\cdots
$$

We notice that the three terms of the asymptotic series (2.11) were used for the estimate of $q_{n}$. We give the table with the above sequences:

$\begin{array}{ccccc}n & \left|t_{n}-\gamma\right| & \left|s_{n}-\gamma\right| & \left|r_{n, 2}^{3}-\gamma\right| & \left|q_{n}-\gamma\right| \\ 250 & 1.30935 \times 10^{-17} & 4.26667 \times 10^{-12} & 2.25298 \times 10^{-14} & 2.03175 \times 10^{-18} \\ 500 & 2.04586 \times 10^{-19} & 2.66667 \times 10^{-13} & 7.07570 \times 10^{-16} & 3.1746 \times 10^{-20} \\ 1000 & 3.19665 \times 10^{-21} & 1.66667 \times 10^{-14} & 2.21668 \times 10^{-17} & 4.96032 \times 10^{-22} \\ 10000 & 3.19665 \times 10^{-27} & 1.66667 \times 10^{-18} & 2.22167 \times 10^{-22} & 4.96032 \times 10^{-28} \\ 50000 & 2.04586 \times 10^{-31} & 2.66667 \times 10^{-21} & 7.11076 \times 10^{-26} & 3.1746 \times 10^{-32}\end{array}$

Using the values from the above table, we conclude the superiority of the sequence $\left(q_{n}\right)_{n \geq 225}$ over Mortici's sequence $\left(t_{n}\right)_{n \geq 225}$, Lu's sequence $\left(r_{n, 2}^{3}\right)_{n \geq 225}$, Cristea and Mortici's sequence $\left(s_{n}\right)_{n \geq 225}$.

Acknowledgements. The author thanks the editors and the anonymous refree for useful ideas which improved much the initial form of this paper. Some computations made in this paper were performed using Maple software.

\section{REFERENCES}

[1] M. Abramowitz, I. A. Stegun: Handbook of Mathematical Functions with Formulas, Graphs, and Mathematical Tables, New York: Dover Publications, 1972.

[2] G. D. Anderson, R. W. Barnard, M. K. Vamanamurthy and M. Vuorinen: Inequalities for zero-balanced hypergeometric functions, Trans. Amer. Math. Soc. 347, (1995), 1713-1723.

[3] C. P. Chen, L. Lin: Remarks on asymptotic expansions for the gamma function, Appl. Math. Lett. 25 (2012), $2322-2326$. 
[4] V. G. Cristea: Asymptotic series and estimates of a convergence to Euler-Mascheroni constant, J. Sci. Arts, 14 (2) (27) (2014), 143-146.

[5] V. G. Cristea, C. Mortici: Latter research on Euler-Mascheroni constant, Contemp. Anal. Appl. Math. 2 (1) (2014), $108-115$.

[6] D. W. DeTemple:A quicker convergences to Euler's constant, Amer. Math. Monthly, 100 (5) (1993) 468-470.

[7] D. W. DeTemple: A geometric look at sequences that convergence to Euler's constant, College Math. J., 37 (2006) 128-131.

[8] R. Graham, D. Knuth and O. Patashnik: Binomial Coefficients. Concrete Mathematics (2nd ed.). Addison Wesley. 153-256, 1994, ISBN 0-201-55802-5.

[9] X. Hu, D. Lu and X. Wang: New Quicker Sequences and Inequalities with Continued Fraction Towards Euler's Constant, Results Math, 73 (1) (2018), 1-10.

[10] E. A. Karatsuba: On the computation of the Euler constant $\gamma$. Computational methods from rational approximation theory (Wilrijk, 1990), Numer. Algorithms 24 (1-2), (2000), 83-97.

[11] C. Mortici: Product approximation via asymptotic integration, Amer. Math. Monthly 117 (5) (2010), 434-441.

[12] C. Mortici: Optimizing the rate of convergence in some new classes of sequences convergent to Euler's constant, Anal. Appl. (Singap.) 8 (1) (2010), 99-107.

[13] C. Mortici: Improved convergence towards generalized Euler-Mascheroni constant, Appl. Math. Comput. 215 (9) (2010), 3443-3448.

[14] C. Mortici: Fast convergence towards Euler-Mascheroni constant, Comp. Appl. Math. Vol. 29, (3) (2010), 479-491.

[15] C. Mortici, A. Vernescu: An improvement of the convergence speed of the sequence $\left(\gamma_{n}\right)_{n \geq 1}$ converging to Euler's constant, An. Şt. Univ. Ovidius Constanţa, 13 (1) (2005), 97-100.

[16] C. Mortici, A. Vernescu: Some new facts in discrete asymptotic analysis, Math. Balkanika, 21 (3-4) (2007), 301-308.

[17] A. Sîntămărian: A generalization of Euler's constant, Numer. Algorithms 46 (2) (2007), 141-151.

[18] S. R. Tims, J. A. Tyrrell: Approximate evaluation of Euler's constant, Math. Gaz. 55 (391) (1971) 65-67.

[19] R. M. Young: Euler's constant, Math. Gaz. 75 (472) (1991) 187-190.

Master MSC. In Didactic Mathematics

VALAHIA UNIVERSITY OF TÂRGOVIŞTE

FACULTY OF SCIENCE AND ARTS

StR. AleEA SinAiA, NR. 13, COD POSTAL 130004, TARGOVISTE

ORCID: 0000-0002-9230-425X

Email address: valentingabrielc@yahoo.com 